\title{
Energy of measures on compact Riemannian manifolds
}

\author{
by \\ Kathryn E. Hare (Waterloo, ON) and \\ MARIA RoginskaYA (Göteborg)
}

\begin{abstract}
We investigate the energy of measures (both positive and signed) on compact Riemannian manifolds. A formula is given relating the energy integral of a positive measure with the projections of the measure onto the eigenspaces of the Laplacian. This formula is analogous to the classical formula comparing the energy of a measure in Euclidean space with a weighted $L^{2}$ norm of its Fourier transform. We show that the boundedness of a modified energy integral for signed measures gives bounds on the Hausdorff dimension of the measure. Refined energy integrals and Hausdorff dimensions are also studied and applied to investigate the singularity of Riesz product measures of dimension one.
\end{abstract}

1. Introduction. In this paper we study the energy of measures on compact, connected Riemannian manifolds. There is a well known and useful formula relating the energy of a positive, finite measure on $\mathbb{R}^{n}$ to a weighted $L^{2}$ norm of its Fourier transform (cf. [16, p. 162]). We obtain an analogous formula comparing the energy of a positive measure on a compact manifold with a weighted sum of the $L^{2}$ norms of the projections of the measure onto the spaces of eigenfunctions of the Laplacian operator. This weighted sum can be thought of as a weighted harmonic representation. The corresponding formulas for measures on the $n$-dimensional sphere or torus, which were obtained in [8] and [9], can be derived as special cases of our new formula.

Even on Euclidean space the natural extension of the energy integral to signed measures need not be well defined. Motivated by [10], we consider a modification of this integral. Our modified integral is defined for all finite measures and is comparable to the usual energy integral in the case of a positive measure. We show that the finiteness of the modified energy

2000 Mathematics Subject Classification: Primary 58C35, 28A12; Secondary 42A55, $28 \mathrm{~A} 78$.

Key words and phrases: energy, Hausdorff dimension, signed measure, Riesz product.

This research was supported in part by NSERC. 
integral gives a lower bound on the Hausdorff dimension of the measure, as was shown in [10] for the energy integral of signed measures on Euclidean spaces.

Measures can be classified by their Hausdorff dimension, the least dimension of any Borel set on which they are concentrated. However, there are many interesting measures which have full Hausdorff dimension but are not absolutely continuous with respect to the Hausdorff measure of that dimension. By introducing refined dimensions based on the functions $x^{n}|\log x|^{s}$ (where $n$ equals the dimension of the manifold), rather than the usual functions $x^{t}, t \leq n$, we are able to quantify the singularity of (some) measures of dimension $n$. We prove that the corresponding refined energy integral can also be compared with a (suitable) weighted harmonic representation and that the finiteness of this integral provides an upper bound on the (appropriate) refined Hausdorff co-dimension.

Our formula relating the refined energy integral with a weighted harmonic representation of the measure is applied to study Riesz products on the torus which have Hausdorff dimension one.

The paper is organized as follows: In Section 2 we derive our analogue of the classical formula comparing the energy of a measure with the size of its Fourier transform. The formula is applied to study the dimensions of central measures on compact Lie groups in Section 3. The modified energy integral for signed measures and its connection with Hausdorff dimension are investigated in Section 4. This involves obtaining a variation of the Besicovitch covering lemma for these manifolds. In Section 5 we study the refined energy integral and refined Hausdorff co-dimensions, and in Section 6 apply these ideas to Riesz product measures on the torus.

\section{Energy of a positive measure and its harmonic representa-} tion. Throughout the paper $M$ will denote a connected, compact Riemannian manifold of dimension $n$. The notation $B(x, r)$ will denote the ball in $M$ centred at $x$ and having radius $r$, and $|B(x, r)|$ will denote its volume.

For a finite, positive, Borel measure $\mu$ on a manifold $M$ one can define the Riesz t-energy of $\mu$ by the formula

$$
I_{t}(\mu)=\int_{M} \int_{M} \frac{1}{d(x, y)^{t}} d \mu(x) d \mu(y)
$$

where $d(x, y)$ is the metric on $M$. As both the kernel and the measure are positive, the integral is well defined and the energy takes values in $[0, \infty]$.

The energy of a measure on $\mathbb{R}^{n}$ has proven to be a useful concept. For example, if $I_{t}(\mu)<\infty$, then the Hausdorff dimension of any set on which $\mu$ is concentrated is at least $t$. There is a well known and important relationship 
between the energy of a measure on $\mathbb{R}^{n}$ and its Fourier transform:

$$
I_{t}(\mu)=c_{t, n} \int|x|^{t-n}|\widehat{\mu}(x)|^{2} d x,
$$

and this has been used to study a variety of things, including the Hausdorff dimension of projections and intersections of sets, distance sets and the average rate of decay of the Fourier transform (cf. [5], [16] and the references cited therein).

The behaviour of the Laplacian, $\Delta f=\operatorname{div}(\nabla f)$, on compact Riemannian manifolds has been thoroughly studied (cf. [3], [4]). The set of eigenvalues of the Laplacian is a discrete sequence $\left\{-\lambda_{k}\right\}$ of non-positive real numbers. The corresponding eigenfunctions are $C^{\infty}$ and can be chosen to form an orthonormal basis in $L^{2}(M)$. The projection of a function $f \in L^{2}(M)$ onto the finite-dimensional eigenspace corresponding to the eigenvalue $-\lambda_{k}$ can be obtained by the formula

$$
P_{k}(f)=\int_{M} K_{k}(x, y) f(y) d y
$$

where $K_{k}(x, y)=\sum_{j} \varphi_{j k}(x) \varphi_{j k}(y)$, the sum being taken over an orthonormal basis, $\left\{\varphi_{j k}\right\}_{j}$, of the eigenspace associated with the eigenvalue $-\lambda_{k}$. The kernel $K_{k}$ is a $C^{\infty}$ function and thus the projection operator $P_{k}$ can be extended to the space of finite measures on $M$. We continue to denote by $P_{k}(\mu)$ the image in $L^{2}$ of this extended operator applied to the measure $\mu$.

The Riesz energy of a measure and its harmonic representation are related by the following formula.

TheOREM 2.1. Let $M$ be a connected, compact, Riemannian manifold of dimension $n$ and suppose $0<t<n$. There exist constants $A, B>0$, depending on $n, t$ and $M$, such that if $\mu$ is a positive, Borel measure on $M$ and $\mu_{k}=P_{k}(\mu)$, then

$$
A I_{n-t}(\mu) \leq \sum_{k}\left(\lambda_{k}+1\right)^{-t / 2}\left\|\mu_{k}\right\|_{2}^{2} \leq B I_{n-t}(\mu) .
$$

The proof of this theorem depends on bounds of the heat kernel, $H(u, x, y)$. By the Sturm-Liouville decomposition (cf. [3, 139-140]) the heat kernel can be represented as

$$
H(u, x, y)=\sum_{k=0}^{\infty} e^{-\lambda_{k} u} K_{k}(x, y),
$$

with the series converging uniformly for each fixed $u>0$. It is well known that the heat kernel is a strictly positive $C^{\infty}$ function on $(0, \infty) \times M \times M$.

As the manifold is compact we may assume that its Ricci curvature is bounded from below. 
Lemma 2.2 (see [14], [15]). For all $\delta>0$ there exist positive constants $c$ and $C$, depending only on $M$ and $\delta$, such that if $x, y \in M$, then

$$
\frac{c \exp \left(-d(x, y)^{2} /(4-\delta) u\right)}{\sqrt{\left|B\left(x, u^{1 / 2}\right)\right|\left|B\left(y, u^{1 / 2}\right)\right|}} \leq H(u, x, y) \leq \frac{C \exp \left(-d(x, y)^{2} /(4+\delta) u\right)}{\sqrt{\left|B\left(x, u^{1 / 2}\right)\right|\left|B\left(y, u^{1 / 2}\right)\right|}}
$$

for all $u>0$ if $M$ has non-negative curvature and for all $u \in(0,1)$ otherwise.

As the volume of any ball of radius $r$ is comparable with $\min \{r, 1\}^{n}$ (cf. $[18,9.1 .6])$, we can reformulate this lemma.

Lemma 2.3. For all $\delta>0$ there exist positive constants $c$ and $C$, depending only on $M$ and $\delta$, such that if $x, y \in M$, then

$$
\begin{aligned}
c(\min \{u, 1\})^{-n / 2} \exp \left(-d(x, y)^{2} /(4-\delta) u\right) \\
\leq H(u, x, y) \leq C(\min \{u, 1\})^{-n / 2} \exp \left(-d(x, y)^{2} /(4+\delta) u\right)
\end{aligned}
$$

for all $u>0$ if $M$ has non-negative curvature and for all $u \in(0,1)$ otherwise.

Proof. We begin by considering $E_{u}(\mu)=\int H(u, x, y) d \mu(x) d \mu(y)$ for $u>0$. Using the Sturm-Liouville decomposition for $H$ we obtain

$$
\int H(u, x, y) d \mu(y)=\sum_{k=0}^{\infty} e^{-u \lambda_{k}} \mu_{k}(x) .
$$

The projection, $\mu_{k}$, is pointwise dominated by $\left\|K_{k}(x, \cdot)\right\|_{\infty}\|\mu\|$. By Weyl's asymptotic formula the number of terms in the sum defining $K_{k}$ is a polynomial in $\lambda_{k}$ and each of the terms is pointwise bounded by a polynomial in $\lambda_{k}$ (see [3, pp. 9, 112]). Thus the series $(2.2)$ is uniformly convergent in $x$ for fixed $u$ and

$$
E_{u}(\mu)=\sum_{k} e^{-u \lambda_{k}} \iint K_{k}(x, y) d \mu(y) d \mu(x)=\sum_{k} e^{-u \lambda_{k}}\left\|\mu_{k}\right\|_{2}^{2} .
$$

Using the estimates of Lemma 2.3 and fixing $\delta=1$ we have

$$
\begin{aligned}
& c \iint(\min \{1, u\})^{-n / 2} \exp \left(-d(x, y)^{2} / 3 u\right) d \mu(x) d \mu(y) \\
& \quad \leq \sum e^{-u \lambda_{k}}\left\|\mu_{k}\right\|_{2}^{2} \leq C \iint(\min \{1, u\})^{-n / 2} \exp \left(-d(x, y)^{2} / 5 u\right) d \mu(x) d \mu(y)
\end{aligned}
$$

for each $u>0$.

If the curvature is non-negative we set $T=\infty$, otherwise take $T=1$. Multiply both sides of the inequalities by the positive function

$$
\psi_{t}(u)= \begin{cases}u^{-1+t / 2} & \text { if } u<1 \\ u^{-1+(t-n) / 2} & \text { if } u \geq 1\end{cases}
$$

and integrate over $u$ in $(0, T)$. If we set

$$
Q_{j}(x, y)=\int_{0}^{T} u^{-1+(t-n) / 2} e^{-d(x, y)^{2} /(4+j) u} d u \quad \text { for } j= \pm 1,
$$


then after changing the order of integration we obtain

$$
\begin{aligned}
\alpha \iint Q_{-1}(x, y) d \mu(x) d \mu(y) & \leq \sum_{k} \int_{0}^{T} \psi_{t}(u) e^{-u \lambda_{k}} d u\left\|\mu_{k}\right\|_{2}^{2} \\
& \leq \beta \iint Q_{1}(x, y) d \mu(x) d \mu(y)
\end{aligned}
$$

for new constants $\alpha, \beta$ which depend only on the dimension. Now

$$
\begin{aligned}
Q_{1}(x, y) & =\left(\frac{d(x, y)^{2}}{5}\right)^{(t-n) / 2} \int_{0}^{T / d(x, y)^{2}} \tau^{-1+(t-n) / 2} e^{-1 / \tau} d \tau \\
& \leq\left(\frac{d(x, y)^{2}}{5}\right)^{(t-n) / 2} \int_{0}^{\infty} \tau^{-1+(t-n) / 2} e^{-1 / \tau} d \tau \\
& =5^{(n-t) / 2} d(x, y)^{t-n} \Gamma\left(\frac{n-t}{2}\right)
\end{aligned}
$$

with equality holding if $T=\infty$. (Here $\Gamma$ is the classical gamma function.) As $M$ is compact, $d(x, y)^{2} \leq(\operatorname{diam} M)^{2}<\infty$ for all $x, y \in M$, and consequently,

$$
\begin{aligned}
Q_{-1}(x, y) & \geq\left(\frac{d(x, y)^{2}}{3}\right)^{(t-n) / 2 T /(\operatorname{diam} M)^{2}} \int_{0} \tau^{-1+(t-n) / 2} e^{-1 / \tau} d \tau \\
& \geq A\left(\frac{d(x, y)^{2}}{3}\right)^{(t-n) / 2}
\end{aligned}
$$

where $A>0$ depends on $n$ and $M$.

When $\lambda_{k}>0$,

$$
\begin{aligned}
\int_{0}^{T} \psi_{t}(u) e^{-u \lambda_{k}} d u & =\int_{0}^{1} u^{-1+t / 2} e^{-u \lambda_{k}} d u+\int_{1}^{T} u^{-1+(t-n) / 2} e^{-u \lambda_{k}} d u \\
& =\lambda_{k}^{-t / 2} \int_{0}^{\lambda_{k}} \tau^{-1+t / 2} e^{-\tau} d \tau+\int_{1}^{T} u^{-1+(t-n) / 2} e^{-u \lambda_{k}} d u .
\end{aligned}
$$

After changing variables the second integral (which is only present if $T=\infty$ ) becomes

$$
\begin{aligned}
\int_{1}^{\infty} u^{-1+(t-n) / 2} e^{-u \lambda_{k}} d u & =\lambda_{k}^{(n-t) / 2} \int_{\lambda_{k}}^{\infty} x^{-1+(t-n) / 2} e^{-x} d x \\
& \leq \lambda_{k}^{-t / 2} \int_{\lambda_{k}}^{\infty} x^{-1+t / 2} e^{-x} d x
\end{aligned}
$$


A routine calculus argument shows that for $x \geq \lambda_{k}$,

$$
x^{-1+t / 2} e^{-x / 2} \leq \begin{cases}(t-2)^{-1+t / 2} e^{-1+t / 2} & \text { if } t>2, \\ \lambda_{1}^{-1+t / 2} e^{-\lambda_{1} / 2} & \text { if } t \leq 2 .\end{cases}
$$

One should note that the minimal non-zero eigenvalue, $\lambda_{1}$, depends only on the manifold. Since $0<t<n$, in either case

$$
x^{-1+t / 2} e^{-x / 2} \leq \sigma
$$

where $\sigma$ is a constant depending on $n$ and the manifold. Thus

$$
\int_{1}^{\infty} u^{-1+(t-n) / 2} e^{-u \lambda_{k}} d u \leq \sigma \lambda_{k}^{-t / 2} \int_{\lambda_{k}}^{\infty} e^{-x / 2} d x \leq 2 \sigma \lambda_{k}^{-t / 2} .
$$

Since the first integral in the final sum of (2.5) dominates

$$
\int_{0}^{\lambda_{1}} \tau^{-1+t / 2} e^{-\tau} d \tau \geq 2 \lambda_{1}^{t / 2} e^{-\lambda_{1}} t^{-1}
$$

we can bound the first term above and below by

$$
\lambda_{k}^{-t / 2} 2 \lambda_{1}^{t / 2} e^{-\lambda_{1}} t^{-1} \leq \lambda_{k}^{-t / 2} \int_{0}^{\lambda_{k}} \tau^{-1+t / 2} e^{-\tau} d \tau \leq \Gamma\left(\frac{t}{2}\right) \lambda_{k}^{-t / 2} .
$$

Note that $\Gamma(t / 2) \sim 1 / t$ when $t$ is close to 0 , hence

$$
\int_{0}^{T} \psi_{t}(u) e^{-u \lambda_{k}} d u \sim \frac{1}{t} \lambda_{k}^{-t / 2}
$$

For $\lambda_{0}=0$, we have

$$
\int_{0}^{\infty} \psi_{t}(u) d u=\frac{2}{t}+\frac{2}{n-t}
$$

Combined with our earlier calculations this implies that for $0<t<n$ we have

$$
\begin{aligned}
\alpha 3^{n-t} I_{n-t}(\mu) & \leq\left(\frac{1}{t}+\frac{1}{n-t}\right)\left\|\mu_{0}\right\|_{2}^{2}+\frac{1}{t} \sum_{k>0} \lambda_{k}{ }^{-t / 2}\left\|\mu_{k}\right\|_{2}^{2} \\
& \leq \beta 5^{n-t} \Gamma\left(\frac{n-t}{2}\right) I_{n-t}(\mu)
\end{aligned}
$$

for new constants $\alpha, \beta$ depending on $n$ and $M$.

We can replace $\lambda_{k}$ with $\lambda_{k}+1$ since the minimal value of $\lambda_{k}$ for $k>0$ depends only on the manifold. This gives the desired formula (2.1).

Corollary 2.4. Let $M$ be a connected, compact manifold of dimension $n$ and $f i x \varepsilon>0$. There exist constants $A, B>0$, depending on $n$ and $\varepsilon$, such 
that if $\mu$ is a positive, Borel measure on $M$ and $\mu_{k}=P_{k}(\mu)$, then for each $0<t<n-\varepsilon$

$$
A I_{n-t}(\mu) \leq \frac{1}{t} \sum_{k}\left(\lambda_{k}+1\right)^{-t / 2}\left\|\mu_{k}\right\|_{2}^{2} \leq B I_{n-t}(\mu) .
$$

Proof. This follows from (2.6) since $\Gamma((n-t) / 2)$ is bounded above and below away from zero when $t<n-\varepsilon$.

REMARK 2.1. We thank Prof. Colzani for helpful conversations on an earlier version of this result for manifolds of positive curvature.

Corollary 2.5. (i) Suppose $M=\mathbb{T}^{n}$ and $\varepsilon>0$. There are constants $A, B>0$, depending on $n$ and $\varepsilon$, such that if $\mu$ is a positive, Borel measure on $M$, then for each $0<t<n-\varepsilon$,

$$
A I_{n-t}(\mu) \leq \frac{1}{t}\left(|\widehat{\mu}(0)|^{2}+\sum_{z \in \mathbb{Z}^{n} \backslash\{0\}}|z|^{-t}|\widehat{\mu}(z)|^{2}\right) \leq B I_{n-t}(\mu) .
$$

(ii) Suppose $M=\mathbb{S}^{n}$, the unit sphere in $\mathbb{R}^{n+1}$, and $\varepsilon>0$. There are constants $A, B>0$ such that if $\mu$ is a positive, Borel measure on $M$, then for each $0<t<n-\varepsilon$,

$$
A I_{n-t}(\mu) \leq \frac{1}{t}\left(\left\|\mu_{0}\right\|_{2}^{2}+\sum_{k=1}^{\infty} k^{-t}\left\|\mu_{k}\right\|_{2}^{2}\right) \leq B I_{n-t}(\mu),
$$

where $\mu_{k}$ denotes the projection of $\mu$ onto the space of spherical harmonics of degree $k$.

Proof. These results follow from the fact that in the first case the eigenfunctions of the Laplacian are the exponential functions $\exp (i x \cdot z)$ with corresponding eigenvalues $-|z|^{2}$, and in the second case the eigenfunctions are the spherical harmonics of degree $k$ with eigenvalues $-k(k+n-1)$.

REMARK 2.2. The result for the torus improves the estimates given in [8]. The formula for the sphere can essentially be found in [9].

2.1. Energy and Hausdorff dimension. The Hausdorff dimension of a measure $\mu$ is defined as

$$
\operatorname{dim}_{\mathrm{H}}(\mu)=\inf \left\{\operatorname{dim}_{\mathrm{H}}(E): E \text { is a Borel set with } \mu(E)>0\right\}
$$

and the energy dimension of $\mu$ is defined as

$$
\operatorname{dim}_{\mathrm{e}}(\mu)=\sup \left\{t: I_{t}(\mu)<\infty\right\}
$$

Our results give the following characterization of energy dimension. 
COROLlary 2.6. If $\mu$ is any positive measure on a connected, compact manifold of dimension $n$, then

$$
\operatorname{dim}_{\mathrm{e}}(\mu)=\sup \left\{t: \sum_{\lambda_{k}>0} \lambda_{k}^{(t-n) / 2}\left\|\mu_{k}\right\|_{2}^{2}<\infty\right\} .
$$

Proposition 2.7. If $\mu$ is any positive measure on a connected, compact manifold of dimension $n$, then $\operatorname{dim}_{\mathrm{H}}(\mu) \geq \operatorname{dim}_{\mathrm{e}}(\mu)$.

Proof. This is proved in $[5,4.13]$ for measures on $\mathbb{R}^{n}$. The same proof holds for any metric space.

3. Energy of central measures on compact Lie groups. An example of a connected, compact Riemannian manifold is a connected, simply connected, compact, simple Lie group $G$ (cf. [18, p. 57]). The coordinate functions of the irreducible representations of $G$ are eigenfunctions of the Laplacian and form an orthogonal basis for $L^{2}(G)$. The eigenvalue of the character of the irreducible representation of $G$ with highest weight $\lambda$ is given by

$$
\langle\varrho, \varrho\rangle-\langle\lambda+\varrho, \lambda+\varrho\rangle
$$

where $\varrho$ is half the sum of the positive roots. If we express $\lambda$ in terms of the fundamental dominant weights $\lambda_{i}$ by $\lambda=\sum m_{i} \lambda_{i}$, and put $m_{\lambda}=\max m_{i}$, then the eigenvalue associated with $\lambda$ is comparable to $-m_{\lambda}^{2}$. (See [13].)

A measure $\mu$ on $G$ is called central if it commutes with all other measures under convolution. Central measures are characterized by the property that their Fourier transform satisfies $\widehat{\mu}(\lambda)=c_{\lambda} I_{\operatorname{deg} \lambda}$ for some constants $c_{\lambda}$. (Here $\operatorname{deg} \lambda$ denotes the degree of representation $\lambda$.) Using this notation we can express formula (2.1) as

$$
I_{t}(\mu) \sim \sum_{\lambda} m_{\lambda}^{t-\operatorname{dim} G}(\operatorname{deg} \lambda)^{2}\left|c_{\lambda}\right|^{2}
$$

It is known that if the measure is also continuous, then $c_{\lambda} \rightarrow 0$ as $\operatorname{deg} \lambda \rightarrow \infty$. In particular (see [11], [12]), if $\mu$ is a continuous, central measure supported on a single conjugacy class, then $\left|c_{\lambda}\right| \leq C(\operatorname{deg} \lambda)^{-s}$ where $s=s(G) \leq O(1 / \operatorname{rank} G)$. If $N$ is the number of positive roots of the Lie group, then it follows from the Weyl character formula that $\operatorname{deg} \lambda \leq C m_{\lambda}^{N}$. Consequently,

$$
I_{t}(\mu) \leq C \sum_{\lambda}(\operatorname{deg} \lambda)^{2-2 s+(t-\operatorname{dim} G) / N},
$$

and this sum is known to be finite if the exponent is less than $-\operatorname{rank} G / N$.

An example of a continuous, central measure supported on the conjugacy class $C(g)$ containing $g \in G$ is the orbital measure, $\mu_{g}$, which is defined by 


$$
\int_{G} f d \mu_{g}=\int_{G} f\left(x^{-1} g x\right) d x \quad \text { for all continuous functions } f,
$$

where $d x$ denotes the Haar measure on $G$. As the orbital measure is uniformly distributed on the submanifold $C(g)$, it is the $t$-dimensional Hausdorff measure on $C(g)$ where $t$ is the dimension of $C(g)$. Thus the energy dimension of $\mu_{g}$ coincides with the dimension of the conjugacy class (cf. $\left.[5,4.13]\right)$. Hence

$$
\sum_{\lambda} m_{\lambda}^{t-\operatorname{dim} G}(\operatorname{deg} \lambda)^{2}\left|c_{\lambda}\right|^{2} \begin{cases}<\infty & \text { for } t<\operatorname{dim} C(g), \\ =\infty & \text { for } t>\operatorname{dim} C(g) .\end{cases}
$$

4. The dimension of signed measures. In this section by a measure we mean a signed, regular, Borel measure of finite variation on the compact, connected Riemannian manifold $M$ of dimension $n$.

The Hausdorff dimension of a signed measure $\mu$ is defined as

$$
\operatorname{dim}_{\mathrm{H}} \mu=\inf \left\{\operatorname{dim}_{\mathrm{H}} E: \mu(E) \neq 0\right\} .
$$

This coincides with the Hausdorff dimension of its total variation $|\mu|$.

We continue to denote by $H(x, z, y)$ the heat kernel on the manifold $M$ and let $\psi_{t}$ be the function defined in (2.3). Put

$$
L_{t}(x, y)=\int \psi_{t}(u) H(u, x, y) d u .
$$

Note that $L_{t}(x, y)$ is comparable to $d(x, y)^{t-n}$. For $0<t<n$ we define the generalized $t$-energy of a signed measure $\mu$ by

$$
I_{t}^{*}(\mu)=\lim _{s \rightarrow 0} \iiint H(s, z, y) L_{n-t}(x, z) d z d \mu(x) d \mu(y) .
$$

For any fixed $s>0$ the heat kernel, $H(s, z, y)$, is a continuous function. Also, $z \mapsto L_{t}(x, z)$ is summable, thus the inner integral is a continuous function of $y$.

As in Section 2 we let $K_{k}$ denote the kernel is of the projection operator onto the eigenspace of the Laplacian corresponding to the eigenvalue $-\lambda_{k}$. An orthogonality argument shows that

$$
\int H(s, z, y) L_{t}(x, z) d z
$$

can be presented by a uniformly convergent series, $\sum c_{j}(t) e^{-s \lambda_{j}} K_{j}(x, y)$, with coefficients

$$
c_{j}(t)=\int \psi_{t}(u) e^{-u \lambda_{j}} d u .
$$

Note that $c_{j}(t) \geq 0$. Thus for any finite measure $\mu$,

$$
\iint\left(\int H(s, z, y) L_{t}(x, z) d z\right) d \mu(x) d \mu(y)=\sum_{j} c_{j}(t) e^{-s \lambda_{j}}\left\|\mu_{j}\right\|_{2}^{2}
$$


This shows that the generalized energy is well defined. Indeed, as $c_{j} \geq 0$ we have

$$
I_{n-t}^{*}(\mu)=\lim _{s \rightarrow 0} \sum_{j} c_{j}(t) e^{-s \lambda_{j}}\left\|\mu_{j}\right\|_{2}^{2}=\sum_{j} c_{j}(t)\left\|\mu_{j}\right\|_{2}^{2} .
$$

Moreover, the proof of Theorem 2.1 shows that this is equivalent to the classical Riesz $(n-t)$-energy when $\mu$ is a positive measure.

We should note that $I_{t}(\mu)<\infty$ does not imply $I_{t}(|\mu|)<\infty$, even in the classical case (see [10]). Even so, we will show that the energy of a signed measure can be used to obtain lower bounds on the Hausdorff dimension of the measure, as was noted in Proposition 2.7 for positive measures. Our methods are similar to those used for measures in Euclidean space in [10]. The main new ingredient is a variation of the Besicovitch covering lemma valid for compact Riemannian manifolds (Lemma 4.2 below).

LEMma 4.1. There is a positive integer $k(n)$ and real number $\Delta=\Delta(M)$ $>0$ such that if $a_{1}, \ldots, a_{k}$ belong to $M$ and $r_{1}, \ldots, r_{k} \in(0, \Delta)$ are given with

$$
a_{i} \notin B\left(a_{j}, r_{j}\right) \quad \text { for } j \neq i, \quad \text { and } \bigcap_{i=1}^{k} B\left(a_{i}, r_{i}\right) \neq \emptyset,
$$

then $k \leq k(n)$.

Proof. Choose $k(n)$ so that there are no more than $k(n)$ vectors in $n$ dimensional Euclidean space, having interior angles greater than $\pi / 4$ to each other.

Now consider a collection of balls as above and choose a point $O$ in the intersection. Since the manifold is complete there exists a segment $\sigma\left(O, a_{i}\right)$ connecting $O$ with the centre of each of the balls ([18, 5.7.2]). Since $O \in$ $B\left(a_{i}, r_{i}\right)$ the length of the segment $\sigma\left(O, a_{i}\right)$ is no more than $r_{i}$. Similarly, there is a segment $\sigma\left(a_{i}, a_{j}\right)$ joining $a_{i}$ and $a_{j}$, and as each ball $B\left(a_{k}, r_{k}\right)$ does not contain any other's centre, the length of this segment is greater than both $r_{i}$ and $r_{j}$. These segments give a triangle on the manifold.

As $M$ is compact and smooth we may assume the sectional curvature is bounded from below. Upon rescaling the metric we can assume the manifold has curvature at least -1 . By $[18,11.2 .1]$ there is a comparison triangle (i.e. with the same side lengths) in $S_{-1}^{n}$, the $n$-dimensional space of constant curvature -1 . The law of cosines (see $[18,11.2 .3]$ ) states that any triangle in $S_{-1}^{n}$ with side lengths $a, b, c$ and angle $\alpha$ opposite $a$ satisfies

$$
\cosh a=\cosh b \cosh c-2 \sinh b \sinh c \cos \alpha .
$$

It is easy to see that if $c \leq b \leq a$ then

$$
\cos \alpha \leq 2 e^{c} \cosh b /\left(e^{c}+1\right)^{2} .
$$

As the right side of the inequality tends to $1 / 2$ as $b, c \rightarrow 0$, it follows that for 
sufficiently small triangles $\alpha \geq \pi / 4$. By Toponogov's Theorem ([18, 11.2.2]) the angles of the original triangle are larger than the angles of those of the comparison triangle and hence are also at least $\pi / 4$. But these angles correspond to the angles between tangent vectors to the segments in the tangent space at the point $O$. Since there can be at most $k(n)$ such vectors, and each ball is associated with such a vector, $k \leq k(n)$.

Using this lemma one can derive the following variation of the Besicovitch covering lemma. Just imitate the proof found in [16, pp. 29-33], replacing Lemma 2.6 of that proof by the lemma above.

Lemma 4.2. Let $A \subseteq M$ and suppose $\mathcal{B}$ is a family of closed balls of radii less than $\Delta$ such that each point of $A$ is the centre of some ball of $\mathcal{B}$. There is a finite or countable collection of balls $B_{i} \in \mathcal{B}$ which cover $A$ and every point of $M$ belongs to at most $k(n)$ balls.

What we will actually need is the following consequence of the covering lemma.

Lemma 4.3. Let $\mu_{1}$ and $\mu_{2}$ be two finite, positive, regular, mutually singular measures on $M$. For any constants $C, c, \varepsilon>0$ there exists a Borel set $K=K(C, c, \varepsilon)$ such that $\mu_{1}(M \backslash K)<\varepsilon$ and $c \mu_{1}(B(\xi, \tau)) \geq C \mu_{2}(B(\xi, \tau))$ for all $\xi \in K$ and $\tau \leq \varrho=\varrho(\varepsilon)$.

Proof. As the measures are mutually singular we can choose two disjoint sets $A_{1}$ and $A_{2}$ such that $\mu_{j}\left(M \backslash A_{j}\right)=0$ for $j=1,2$. Choose two compact sets $K_{1}$ and $K_{2}$ such that $K_{j} \subset A_{j}$ and $\mu_{j}\left(M \backslash K_{j}\right)<c^{\prime} \varepsilon$, where the constant $c^{\prime}$ depends on $c, C$ and $n$ and will be specified later. Let $\varrho=$ $\min \left(\Delta, \frac{1}{2} \operatorname{dist}\left(K_{1}, K_{2}\right)\right)$.

Let us denote by $K^{\prime}$ the Borel set

$$
K^{\prime}=\left\{\xi \in K_{1}: c \mu_{1}(B(\xi, \tau))<C \mu_{2}(B(\xi, \tau)) \text { for some } \tau \leq \varrho\right\} .
$$

We wish to estimate $\mu_{1}\left(K^{\prime}\right)$. By definition, for each point $x \in K^{\prime}$ there exists a ball $B_{x}$ centred at $x$ of radius less than $\Delta$ which does not intersect $K_{2}$ and for which $c \mu_{1}\left(B_{x}\right)<C \mu_{2}\left(B_{x}\right)$. By the version of the Besicovitch covering lemma proved above we can choose a covering $\left\{B_{k}\right\}$ of $K^{\prime}$ by these balls, with the property that each point of $K^{\prime}$ belongs to at most $k(n)$ balls. Then

$$
\mu_{1}\left(K^{\prime}\right) \leq \sum \mu_{1}\left(B_{k}\right) \leq \sum c^{-1} C \mu_{2}\left(B_{k}\right) \leq c^{-1} C k(n) \mu_{2}\left(\bigcup B_{k}\right) .
$$

Since the balls $B_{k}$ are disjoint from $K_{2}$ we obtain

$$
\mu_{1}\left(K^{\prime}\right) \leq c^{-1} C k(n) \mu_{2}\left(M \backslash K_{2}\right)<c^{-1} C k(n) c^{\prime} \varepsilon .
$$

If we choose $c^{\prime}=\min (1 / 2, c / 2 C k(n))$, then the set $K=K_{1} \backslash K^{\prime}$ satisfies the required conditions.

There is one additional technical result we need. 
Lemma 4.4. Let $0<t<n$. There exist constants $A, B$, depending only on the manifold and $t$, such that for any small $s>0$ there is a positive, piecewise smooth, decreasing function $F_{s, t}$ satisfying

$$
\begin{aligned}
A F_{s, t}(d(x, y)) & \leq \int L_{n-t}(x, z) H(s, z, y) d z \sim \int d(x, z)^{-t} H(s, z, y) d z \\
& \leq B F_{s, t}(d(x, y)) .
\end{aligned}
$$

Proof. The function which we will see works is

$$
F_{s, t}(\tau)=\min \left\{\beta_{1} s^{-t / 2}, \beta_{2} \tau^{-t}\right\}
$$

for a suitable choice of $\beta_{1}, \beta_{2}$.

CASE 1: $d(x, y)<\sqrt{s}$. Divide the area of integration into the ball $B(x, c \sqrt{s})$ and the remainder of the manifold. Since the volume of a small ball in $M$ is comparable to the volume of the corresponding ball in $\mathbb{R}^{n}$, Lemma 2.2 implies that inside $B(x, c \sqrt{s})$ the heat kernel can be estimated above and below by $c s^{-n / 2}$ (for $s<1$ ). Thus the integral is comparable to

$$
(c \sqrt{s})^{-n} \int_{|y| \leq c \sqrt{s}}|y|^{-t} d y=c_{1} s^{-t / 2} .
$$

Outside the ball the function $|\cdot|^{-t}$ is dominated by $(c \sqrt{s})^{-t}$. Also, the integral of the heat kernel is less than the integral over the whole sphere, which is one. Thus the right side estimate is $B s^{-t / 2}$ and the left estimate is $A s^{-t / 2}$.

CASE 2: $d(x, y) \geq \sqrt{s}$. We consider two balls: $B(x, d(x, y) / 2)$ and $B(y, d(x, y) / 2)$. For $z$ in the first ball the distance between $y$ and $z$ varies between $d(x, y) / 2$ and $3 d(x, y) / 2$. Thus the heat kernel on the first ball can be estimated below and above (for short time) by $s^{-n / 2} e^{-27 d(x, y)^{2} / 4 s}$ and $s^{-n / 2} e^{-d(x, y)^{2} / 20 s}$ respectively. Hence

$$
\begin{array}{rl}
A e^{-27 d(x, y)^{2} / 4 s}\left(\frac{d(x, y)^{2}}{s}\right)^{n / 2} & d(x, y)^{-t} \\
\leq & \int_{B(x, d(x, y) / 2)} d(x, z)^{-t} H(s, z, y) d z \\
\leq & B e^{-d(x, y)^{2} / 20 s}\left(\frac{d(x, y)^{2}}{s}\right)^{n / 2} d(x, y)^{-t} .
\end{array}
$$

For $z$ in the second ball,

$$
\frac{1}{2} d(x, y) \leq d(z, x) \leq \frac{3}{2} d(x, y),
$$

and thus $L_{n-t}(x, z)$ is equivalent to $d(x, y)^{-t}$. As this ball contains the ball of radius $c \sqrt{s}$, on which the heat kernel is greater than $c_{2} s^{-n / 2}$, the integral of the heat kernel is at least $c_{0}$ and at most 1 . Thus the integral of the product over the second ball is equivalent to $d(x, y)^{-t}$. The integral over 
the rest of the space is positive and less than $(d(x, y) / 2)^{-t}$, and therefore is inconsequential. To complete this case we simply remark that the functions

$$
e^{-d(x, y)^{2} / k s}\left(\frac{d(x, y)^{2}}{s}\right)^{n / 2} d(x, y)^{-t}+d(x, y)^{-t},
$$

for any fixed $k>0$, are equivalent to $d(x, y)^{-t}$ as $d(x, y)^{2} \geq s$.

It is clear that $F_{s, t}$ is decreasing.

We are now ready to prove the main result of this section.

ThEOREM 4.5. Suppose $\mu$ is a non-zero signed measure on $M$ and $I_{t}^{*}(\mu)$ $<\infty$. Then $\operatorname{dim}_{\mathrm{H}}(\mu) \geq t$.

Proof. To prove this we will approximate $\mu_{+}$and $\mu_{-}$in the strong sense by positive measures with finite $t$-energy. As the approximation is constructed in same way for $\mu_{+}$and $\mu_{-}$, we give the proof only for $\mu_{+}$.

Let $K=K(C, c, \varepsilon)$ be the set given by Lemma 4.3 with the singular measures being $\mu_{+}$and $\mu_{-}$and with $c=A / 2, C=2 B$ ( $A, B$ as in Lemma 4.4) and arbitrary $\varepsilon>0$. Let $\varrho=\varrho(\varepsilon)$. Denote $\left.\mu_{+}\right|_{K}$ by $\mu_{\varrho}$ and let $\mu_{r}=$ $\mu_{+}-\mu_{\varrho}$. For small $s>0$ let

$$
\theta(x, y) \equiv \theta_{s, t}(x, y) \equiv \int_{M} L_{n-t}(x, z) H(s, z, y) d z .
$$

Since $I_{t}^{*}(\mu)<\infty$, for $s$ near zero there is some fixed number $\Theta$ such that

$$
\begin{aligned}
\Theta & \geq \int \theta(x, y) d \mu(x) d \mu(y) \\
= & \int \theta(x, y)\left(d \mu_{+}(x) d \mu_{+}(y)+d \mu_{-}(x) d \mu_{-}(y)-2 d \mu_{+}(x) d \mu_{-}(y)\right) \\
\geq & \int \theta(x, y)\left(d \mu_{\varrho}(x) d \mu_{+}(y)-2 d \mu_{\varrho}(x) d \mu_{-}(y)\right) \\
& \quad+\int \theta(x, y)\left(d \mu_{r}(x) d \mu_{r}(y)+d \mu_{-}(x) d \mu_{-}(y)-2 d \mu_{r}(x) d \mu_{-}(y)\right) .
\end{aligned}
$$

The final integral in the expression above is equal to

$$
\sum_{j} c_{j} e^{-s \lambda_{j}}\left\|\left(\mu_{r}-\mu_{-}\right)_{j}\right\|_{2}^{2}
$$

where we remind the reader that $c_{j}=c_{j}(n-t)$ was defined in (4.1). As the coefficients $c_{j}$ are non-negative, the final integral is non-negative. So

$$
\begin{aligned}
\Theta \geq & \int \theta(x, y)\left(d \mu_{\varrho}(x) d \mu_{+}(y)-2 d \mu_{\varrho}(x) d \mu_{-}(y)\right) \\
= & \int_{d(x, y) \geq \varrho} \theta(x, y)\left(\left(d \mu_{+}(y)-2 d \mu_{-}(y)\right) d \mu_{\varrho}(x)\right) \\
& +\int_{d(x, y)<\varrho} \theta(x, y)\left(d \mu_{+}(y)-2 d \mu_{-}(y)\right) d \mu_{\varrho}(x) .
\end{aligned}
$$


The explicit formula for $F_{s, t}$ given in the proof of Lemma 4.4 shows that for small enough $s$ the integral over the region $\{(x, y): d(x, y) \geq \varrho\}$ dominates

$$
\int_{d(x, y) \geq \varrho} \theta(x, y) d \mu_{+}(y) d \mu_{\varrho}(x)-2 B \beta_{2}\left\|\mu_{-}\right\|\left\|\mu_{\varrho}\right\| \varrho^{-t} .
$$

The integral over $\{(x, y): d(x, y)<\varrho\}$ can be estimated from below as follows: Lemma 4.4 again shows (writing $F$ for $F_{s, t}$ )

$$
\begin{aligned}
& \int_{d(x, y)<\varrho} \theta(x, y)\left(d \mu_{+}(y)-2 d \mu_{-}(y)\right) \\
& \quad \geq A \int_{d(x, y)<\varrho} F(d(x, y)) d \mu_{+}(y)-2 B \int_{d(x, y)<\varrho} F(d(x, y)) d \mu_{-}(y) .
\end{aligned}
$$

The definition of integral shows that the last expression equals

$$
\begin{aligned}
& A\left(F(\varrho) \mu_{+}\{y: F(d(x, y))>F(\varrho)\}+\int_{F(\varrho)}^{\infty} \mu_{+}\{F(d(x, y))>\omega\} d \omega\right) \\
& -2 B\left(F(\varrho) \mu_{-}\{F(d(x, y))>F(\varrho)\}+\int_{F(\varrho)}^{\infty} \mu_{-}\{F(d(x, y))>\omega\} d \omega\right) .
\end{aligned}
$$

Since $F$ is decreasing, the sets $\{y: F(d(x, y))>\omega\}$ for $\omega>F(\varrho)$ are balls of radii less than $\varrho$. Hence the choice of $K$ ensures that

$$
\frac{A}{2} \mu_{+}\{y: F(d(x, y))>\omega\} \geq 2 B \mu_{-}\{F(d(x, y))>\omega\}
$$

for any $x \in K$. Thus for $\mu_{\varrho}$-a.e. $x$ expression (4.3) dominates

$$
\begin{aligned}
\frac{1}{2} A & \left(F(\varrho) \mu_{+}\{F(d(x, y))>F(\varrho)\}+\int_{F(\varrho)}^{\infty} \mu_{+}\{F(d(x, y))>\omega\} d \omega\right) \\
= & \frac{1}{2} A \int_{d(x, y)<\varrho} F(d(x, y)) d \mu_{+}(y) \geq \frac{1}{2} A B^{-1} \int_{d(x, y)<\varrho} \theta(x, y) d \mu_{+}(y) .
\end{aligned}
$$

Consequently,

$$
\begin{aligned}
\iint_{d(x, y)<\varrho} \theta(x, y)\left(d \mu_{+}(y)-\right. & \left.2 d \mu_{-}(y)\right) d \mu_{\varrho}(x) \\
& \geq \frac{1}{2} A B^{-1} \iint_{d(x, y)<\varrho} \theta(x, y) d \mu_{+}(y) d \mu_{\varrho}(x) .
\end{aligned}
$$

As all the estimates are independent of $s$, and $d \mu_{+} \geq d \mu_{\varrho}$, these arguments imply

$$
\Theta \geq \frac{1}{2} A B^{-1} I_{t}^{*}\left(\mu_{\varrho}\right)-2 B \beta_{2}\left\|\mu_{-}\right\|\left\|\mu_{\varrho}\right\| \varrho^{-t}
$$


and consequently, $I_{t}^{*}\left(\mu_{\varrho}\right)<\infty$. To conclude, note that by construction $\mu_{\varrho} \rightarrow$ $\mu_{+}$in the strong sense as $\varepsilon \rightarrow 0$.

5. Refined Hausdorff and energy dimensions. Many measures have Hausdorff dimension equal to the dimension of the manifold and yet are concentrated on sets which are small, in some sense. For instance, many Riesz product measures on the torus have dimension one but are singular to Lebesgue measure as they are concentrated on sets of Lebesgue measure zero.

In this section we study generalized Hausdorff measures and refined energy integrals based on the functions $x^{n}|\log x|^{s}$ (with $n$ equal to the dimension of the manifold), rather than the usual functions $x^{t}$. These measures and integrals give rise to refined Hausdorff and energy co-dimensions which can be used to quantify the singularity of (some) measures of maximum Hausdorff dimension.

We will obtain formulas for the refined energy integrals analogous to (2.1). The finiteness of these refined energy integrals will be seen to give an upper bound on the corresponding refined Hausdorff co-dimensions.

5.1. Definitions and basic properties of refined dimensions. Suppose $h$ : $[0, \infty) \rightarrow[0, \infty]$ is a right continuous function which is increasing near zero and satisfies $h(0)=0$. We define the $h$-Hausdorff measure of a Borel set $E$ in a metric space $X$ by (see [2], [20])

$$
h_{\delta}(E)=\inf \left\{\sum h\left(\operatorname{diam} U_{j}\right): \bigcup U_{j} \supseteq F, \operatorname{diam} U_{j} \leq \delta\right\}
$$

and

$$
h(E)=\lim _{\delta \rightarrow 0} h_{\delta}(E) .
$$

Of course, the special case when $h(x)=x^{t}$ is the usual $t$-dimensional Hausdorff measure.

Similarly, we can define the h-energy of a finite, regular, Borel measure on $X$ by

$$
I_{h}(\mu)=\iint \frac{d \mu(x) d \mu(y)}{h(d(x, y))} .
$$

When $h(x)=x^{t}$ we simply write $I_{t}(\mu)$ for the usual $t$-energy of $\mu$.

It is natural to assume $h$ satisfies the doubling condition, i.e., there is a constant $C$ such that $h(2 r) \leq C h(r)$ for all $r>0$. Under this assumption the following relationships hold.

Proposition 5.1. Suppose $\mu$ is a measure on $X$ and $F \subseteq X$ is a Borel set. 
(i) There is a constant $A$ such that if

$$
\limsup _{r \rightarrow 0} \mu(B(x, r)) / h(2 r)<c<\infty
$$

for all $x \in F$, then $h(F) \geq A \mu(F) / c$.

(ii) Suppose $X=\mathbb{R}^{n}$. There is a constant $B$ such that if

$$
\limsup _{r \rightarrow 0} \mu(B(x, r)) / h(2 r)>c>0
$$

for all $x \in F$, then $h(F) \leq B \mu\left(\mathbb{R}^{n}\right) / c$.

Proposition 5.2. If there exists a measure $\mu$ on $F \subseteq X$ such that $I_{h}(\mu)<\infty$, then $h(F)=\infty$.

The proofs of both these results are analogous to those given in [5, p. 61] for $t$-dimensional Hausdorff measure and $t$-energy.

Now suppose the metric space $X$ is a compact manifold $M$. We will write $\varrho=\varrho(M)$ for twice the diameter of $M$. As $M$ is compact, $\varrho<\infty$.

Our main interest is in the special case when

$$
h_{n, s}(x)=x^{n}|\log (x / \varrho)|^{s} \quad \text { for } n=\operatorname{dim} M \text { and } s \geq 0 .
$$

This function is continuous (defining $h(0)=0$ ), increasing near zero and satisfies the doubling condition. The measures $h_{n, s}$ are clearly a refinement of $n$-dimensional Hausdorff measure. We will write $I_{n, s}$ for $I_{h_{n, s}}$.

For small $x, x^{n}|\log (x / \varrho)|^{s}$ increases as $s$ increases, thus (for fixed $n$ ) the measures $h_{n, s}(F)$ also increase as $s$ increases. It easily follows that if $s<t$ and $h_{n, t}(F)<\infty$, then $h_{n, s}(F)=0$. Thus it seems natural to consider the functions $h_{n, s}(F)$ as measuring the co-dimension of $F$ and we define the fine $n$-Hausdorff co-dimension of a subset $E \subseteq M$ of Hausdorff dimension $n$ as

$$
\text { Fine }_{n} \operatorname{dim}_{H}(F) \equiv \sup \left\{s: h_{n, s}(F)=0\right\}=\inf \left\{s: h_{n, s}(F)=\infty\right\} \text {. }
$$

Recall (see [6, p. 171]) that the Hausdorff dimension of a measure $\mu$ on $M$ is defined by

$$
\operatorname{dim}_{\mathrm{H}}(\mu) \equiv \inf \left\{\operatorname{dim}_{\mathrm{H}}(F): \mu(F)>0\right\} .
$$

For measures $\mu$ of Hausdorff dimension $n$, we define the fine $n$-Hausdorff co-dimension of $\mu$ as

$$
\text { Fine }_{n} \operatorname{dim}_{\mathrm{H}}(\mu) \equiv \sup \left\{\text { Fine }_{n} \operatorname{dim}_{\mathrm{H}}(F): \mu(F)>0\right\} .
$$

The energy dimension of $\mu$ is given by

$$
\operatorname{dim}_{\mathrm{e}}(\mu) \equiv \inf \left\{s: I_{s}(\mu)=\infty\right\}=\sup \left\{s: I_{s}(\mu)<\infty\right\} .
$$

Analogously, we define the fine n-energy co-dimension by

$$
\text { Fine }_{n} \operatorname{dim}_{\mathrm{e}}(\mu) \equiv \inf \left\{s: I_{n, s}(\mu)<\infty\right\}=\sup \left\{s: I_{n, s}(\mu)=\infty\right\} .
$$

With this notation the propositions yield the following corollaries: 
Corollary 5.3. (i) If $\lim \sup _{r \rightarrow 0} \mu(B(x, r)) / h_{n, s}(2 r)<c$ for all $x \in$ $F \subseteq X$, then the fine $n$-Hausdorff co-dimension of $F$ is bounded above by $s$.

(ii) If $\limsup _{r \rightarrow 0} \mu(B(x, r)) / h_{n, s}(2 r)>c>0$ for all $x \in F \subseteq \mathbb{R}^{n}$, then the fine $n$-Hausdorff co-dimension of $F$ is bounded below by s.

Corollary 5.4. If there exists a measure $\mu$ on $F$ such that $I_{n, s}(\mu)<\infty$, then Fine $_{n} \operatorname{dim}_{\mathrm{H}}(F) \leq s$. Consequently,

$$
\text { Fine }_{n} \operatorname{dim}_{\mathrm{H}}(\mu) \leq \text { Fine }_{n} \operatorname{dim}_{\mathrm{e}}(\mu) .
$$

There is also a partial converse to this result.

Proposition 5.5. Suppose there exists a constant $c$ and $r_{0}>0$ such that $\mu(B(x, r)) \leq$ ch $_{n, s}(r)$ for $\mu$ a.e. $x$ and $r \leq r_{0}$. Then $I_{n, t}(\mu)<\infty$ for all $t>s+1$.

Proof. Set $\omega(r)=\mu(B(x, r))$. Let

$$
\phi_{t}(y) \equiv \int_{d(x, y) \leq r_{0}} \frac{d \mu(x)}{d(x, y)^{n}|\log (d(x, y) / \varrho)|^{t}}=\int_{0}^{r_{0}} r^{-n}|\log (r / \varrho)|^{-t} d \omega(r) .
$$

Upon integrating by parts it follows that

$$
\begin{aligned}
\phi_{t}(y) & =\left.r^{-n}\left|\log \frac{r}{\varrho}\right|^{-t} \omega(r)\right|_{0} ^{r_{0}}+\int_{0}^{r_{0}} \frac{n \omega(r)}{r^{n+1}}\left|\log \frac{r}{\varrho}\right|^{-t}\left(1-\frac{t}{n}\left|\log \frac{r}{\varrho}\right|^{-1}\right) d r \\
& \leq C_{1}+\int_{0}^{r_{0}} n r^{-1}\left|\log \frac{r}{\varrho}\right|^{s-t}\left(1-\frac{t}{n}\left|\log \frac{r}{\varrho}\right|^{-1}\right) d r,
\end{aligned}
$$

and this is finite as $t>s+1$. Since

$$
\iint_{d(x, y) \geq r_{0}} \frac{d \mu(x) d \mu(y)}{d(x, y)^{n}|\log (d(x, y) / \varrho)|^{t}}=C\left(r_{0}\right)<\infty,
$$

it follows that for $t>s+1$,

$$
I_{n, t}(\mu) \leq C\left(r_{0}\right)+\int \phi_{t}(y) d \mu(y)<\infty .
$$

5.2. The refined energy integral and its harmonic representation. Next, we show how to use formula (2.1) to obtain a similar formula for the refined energy integral. We continue to use the notation of Section 2.

TheOREM 5.6. Let $M$ be a connected, compact, Riemannian manifold of dimension $n$. Suppose $s>1$. There are constants $A, B>0$, depending on $M$ and $n$, such that if $\mu$ is any finite, regular, Borel measure on $M$ with energy dimension $n$ and $\mu_{k}=P_{k}(\mu)$, then the refined energy integral, $I_{n, s}$, satisfies

$$
A I_{n, s}(\mu) \leq\left\|\mu_{0}\right\|_{2}^{2}+\sum_{\lambda_{k} \neq 0}\left(\log \left|\lambda_{k}+1\right|\right)^{1-s}\left\|\mu_{k}\right\|_{2}^{2} \leq B I_{n, s}(\mu) .
$$


Proof. We can rewrite the estimates of (2.1) (see Cor. 2.4), with different constants, in a form more useful for our arguments: for $0<t<1 / 2$,

$$
\begin{aligned}
A \iint \frac{d \mu(x) d \mu(y)}{(d(x, y) / \varrho)^{n-t}} & \leq \frac{1}{t}\left(\left\|\mu_{0}\right\|_{2}^{2}+\sum_{\lambda_{k} \neq 0}\left(\lambda_{k}+1\right)^{-t / 2}\left\|\mu_{k}\right\|_{2}^{2}\right) \\
& \leq B \iint \frac{d \mu(x) d \mu(y)}{(d(x, y) / \varrho)^{n-t}} .
\end{aligned}
$$

Multiply through by $t^{\alpha}$ with $\alpha=s-1$ and integrate over $t \in[0,1 / 2]$. After invoking Fubini's theorem and changing variables, the inner integral (for the two outside terms in the inequality) simplifies to

$$
\begin{aligned}
\int_{0}^{1 / 2} \frac{\varrho^{n-t} t^{\alpha}}{d(x, y)^{n-t}} d t & =\frac{\varrho^{n}}{d(x, y)^{n}} \int_{0}^{1 / 2} t^{\alpha}\left(\frac{d(x, y)}{\varrho}\right)^{t} d t \\
& =\frac{\varrho^{n}}{d(x, y)^{n}|\log (d(x, y) / \varrho)|^{\alpha+1}} \int_{0}^{\frac{1}{2}|\log (d(x, y) / \varrho)|} \tau^{\alpha} e^{-\tau} d \tau .
\end{aligned}
$$

Since $2 d(x, y) \leq \varrho<\infty,|\log (d(x, y) / \varrho)|$ is finite and bounded away from zero. Hence

$$
\int_{0}^{1 / 2} \frac{\varrho^{n-t} t^{\alpha}}{d(x, y)^{n-t}} d t \sim \frac{\varrho^{n}}{d(x, y)^{n}|\log (d(x, y) / \varrho)|^{\alpha+1}} .
$$

As the eigenvalues of the Laplacian are discrete there is some $C>1$ such that $\log \left(\lambda_{k}+1\right) \geq \log C$ for all $\lambda_{k} \neq 0$. Thus

$$
\begin{aligned}
\int_{0}^{1 / 2} t^{\alpha-1}\left(\lambda_{k}+1\right)^{-t / 2} d t & =\frac{2}{\left(\log \left(\lambda_{k}+1\right)\right)^{\alpha}} \int_{0}^{\frac{1}{4} \log \left(\lambda_{k}+1\right)} \tau^{\alpha-1} e^{-\tau} d \tau \\
& \sim \frac{2}{\left(\log \left(\lambda_{k}+1\right)\right)^{\alpha}}
\end{aligned}
$$

Of course, $\int_{0}^{1 / 2} t^{\alpha-1} d t=O(1 / \alpha)$. Together these estimates give

$$
\begin{aligned}
A_{1} \iint \frac{d \mu(x) d \mu(y)}{d(x, y)^{n}|\log (d(x, y) / \varrho)|^{s}} & \leq\left\|\mu_{0}\right\|_{2}^{2}+\sum_{\lambda_{k} \neq 0}\left(\log \left|\lambda_{k}+1\right|\right)^{1-s}\left\|\mu_{k}\right\|_{2}^{2} \\
& \leq B_{1} \iint \frac{d \mu(x) d \mu(y)}{d(x, y)^{n} \mid \log (d(x, y) / \varrho)^{s}}
\end{aligned}
$$

which is the desired result.

If we apply these results to the specific case of measures on an $n$ dimensional torus or sphere, then we obtain the following corollaries. 
Corollary 5.7. (i) If $\mu$ is any measure on $\mathbb{T}^{n}$ of Hausdorff dimension $n$, then

$$
I_{n, s}(\mu) \sim|\widehat{\mu}(0)|^{2}+\sum_{k \in \mathbb{Z}^{n} \backslash\{0\}}(\log (|k|+1))^{1-s}|\widehat{\mu}(k)|^{2} .
$$

(ii) If $\mu$ is any measure on $\mathbb{S}^{n}$, the unit sphere in $\mathbb{R}^{n+1}$, then

$$
I_{n, s}(\mu) \sim\left\|\mu_{0}\right\|_{2}^{2}+\sum_{k=1}^{\infty}(\log |k+1|)^{1-s}\left\|\mu_{k}\right\|_{2}^{2}
$$

where $\mu_{k}$ denotes the projection of $\mu$ onto the space of spherical harmonics of degree $k$.

6. Refined dimension of Riesz products on the torus. A sequence $\left\{\gamma_{k}\right\} \subseteq \mathbb{Z}^{n}$ is called dissociate if for any positive integer $N$,

$$
\sum_{k=1}^{N} \varepsilon_{k} \gamma_{k}=0 \quad \text { for } \varepsilon_{k}=0, \pm 1, \pm 2 \quad \text { implies } \quad \varepsilon_{k}=0 \quad \text { for all } k .
$$

A lacunary sequence $\left\{n_{k}\right\}$ of positive integers with $n_{k+1} / n_{k} \geq 3$ is an example of a dissociate sequence in $\mathbb{Z}$.

Given a dissociate sequence $\left\{\gamma_{k}\right\}$ and a sequence $\left\{a_{k}\right\}$ of complex numbers satisfying $\sup _{k}\left|a_{k}\right| \leq 1$, we define trigonometric polynomials $P_{k}(x)=$ $\prod_{j=1}^{k}\left(1+\operatorname{Re} a_{j} e^{i \pi \gamma_{j} \cdot x}\right)$ for $x \in \mathbb{T}^{n}$. By a Riesz product measure

$$
\mu_{\left\{a_{j}\right\}} \equiv \prod_{j=1}^{\infty}\left(1+\operatorname{Re} a_{j} e^{i \pi \gamma_{j} \cdot x}\right)
$$

we mean the weak* limit of the measures $P_{k}(x) d x$ on $\mathbb{T}^{n}$.

6.1. Refined Hausdorff co-dimension. Estimates of the Hausdorff dimension of Riesz products on $\mathbb{T}$ were first obtained by Peyrière in [19] using probabilistic ideas. He proved that if $\sup _{k}\left|a_{k}\right|<1, n_{k+1} / n_{k} \in \mathbb{Z}$ and $n_{k+1} / n_{k} \geq 3$, then the Hausdorff dimension of the Riesz product measure $\mu_{\left\{a_{j}\right\}}=\prod_{j=1}^{\infty}\left(1+\operatorname{Re} a_{j} e^{i \pi n_{j} x}\right)$ satisfies

$1-\liminf _{k \rightarrow \infty}\left(\frac{\int \log P_{k} d \mu_{\left\{a_{j}\right\}}}{\log n_{k+1}}\right) \geq \operatorname{dim}_{\mathrm{H}}\left(\mu_{\left\{a_{j}\right\}}\right) \geq 1-\limsup _{k \rightarrow \infty}\left(\frac{\int \log P_{k} d \mu_{\left\{a_{j}\right\}}}{\log n_{k}}\right)$ $([19,2.8])$. This implies that the Hausdorff dimension of the Riesz product $\mu_{\left\{a_{j}\right\}}$ is bounded below by

$$
1-\limsup _{k \rightarrow \infty}\left(\sum_{j=1}^{k}\left|a_{j}\right| / \log n_{k}\right)
$$


and when the coefficients are small in modulus it is bounded above by approximately

$$
1-\liminf _{k \rightarrow \infty}\left(\frac{1}{4} \sum_{j=1}^{k}\left|a_{j}\right|^{2} / \log n_{k}\right) .
$$

Of course, if $\left|a_{k}\right| \rightarrow 0$ or $n_{k} \rightarrow \infty$ sufficiently quickly, then the Hausdorff dimension of $\mu_{\left\{a_{j}\right\}}$ is 1 , even though $\mu_{\left\{a_{j}\right\}}$ is a singular measure unless $\left\{a_{j}\right\} \in l^{2}$. This is true, for example, if $k / \log n_{k} \rightarrow 0$ or $n_{k+1} / n_{k} \rightarrow \infty$. For such measures the refined Hausdorff co-dimension is of interest and for certain of these measures there is an analogue of Peyrière's result.

Proposition 6.1. Suppose $\sup _{k}\left|a_{k}\right|<1, n_{k+1} / n_{k} \in \mathbb{Z}, \sum\left(\log \log n_{k}\right)^{-2}$ $<\infty$. Let $\mu=\mu_{\left\{a_{j}\right\}}$ be the Riesz product $\mu=\prod_{j=1}^{\infty}\left(1+\operatorname{Re} a_{j} e^{i \pi n_{j} x}\right)$. Then the refined Hausdorff co-dimension of $\mu$ satisfies

$\liminf _{k \rightarrow \infty}\left(\frac{\int \log P_{k} d \mu_{\left\{a_{j}\right\}}}{\log \log n_{k+1}}\right) \leq$ Fine $_{1} \operatorname{dim}_{\mathrm{H}}\left(\mu_{\left\{a_{j}\right\}}\right) \leq \limsup _{k \rightarrow \infty}\left(\frac{\int \log P_{k} d \mu_{\left\{a_{j}\right\}}}{\log \log n_{k}}\right)$.

Proof. Set

$$
s_{0}=\liminf _{k \rightarrow \infty}\left(\frac{\int \log P_{k} d \mu_{\left\{a_{j}\right\}}}{\log \log n_{k+1}}\right), \quad t_{0}=\limsup _{k \rightarrow \infty}\left(\frac{\int \log P_{k} d \mu_{\left\{a_{j}\right\}}}{\log \log n_{k}}\right) .
$$

Proposition 2.5 of [19] shows that under the assumption $\sum\left(\log \log n_{k}\right)^{-2}$ $<\infty$, the series

$$
\sum\left(\log \log n_{k}\right)^{-1}\left(\log \left(1+\operatorname{Re}\left(a_{k} e^{i \pi n_{k} x}\right)\right)-\int \log \left(1+\operatorname{Re}\left(a_{k} e^{i \pi n_{k} t}\right)\right) d \mu\right)
$$

converges for $\mu$-a.e. $x$. By Kronecker's lemma $([17$, p. 147])

$$
\left(\log \log n_{k}\right)^{-1}\left(\log P_{k}(x)-\int \log P_{k}(t) d \mu\right)=\gamma_{k}(x)
$$

where $\gamma_{k}(x)$ tends to zero for a.e. $x$.

Choose an interval $I$ whose length satisfies $2 / n_{k+1} \leq|I| \leq 1 / n_{k}$. It is easy to see that if $x, t \in I$ then

$$
\left|\log P_{k}(t)-\log P_{k}(x)\right| \leq c
$$

where $c$ is a constant depending on the sequence $\left\{a_{j}\right\}$. If we let $\mu_{k}$ denote the Riesz product $\prod_{j=k+1}^{\infty}\left(1+\operatorname{Re} a_{j} e^{i \pi n_{j} x}\right)$, then $\mu(I)=\int_{I} P_{k}(t) d \mu_{k}$ and therefore is comparable to $P_{k}(x) \mu_{k}(I)$. Peyrière shows that $\mu_{k}(I)$ is comparable to $|I|$ so that $\mu(I) /|I| \sim P_{k}(x)$. Therefore, for a suitable constant $C$,

But

$$
\frac{\log P_{k}(x)-\log C}{\log \log n_{k}} \leq \frac{\log (\mu(I) /|I|)}{\log \log n_{k}} \leq \frac{\log P_{k}(x)+\log C}{\log \log n_{k}} .
$$

$$
\frac{\log P_{k}(x)}{\log \log n_{k}}=\gamma_{k}(x)+\frac{\int \log P_{k} d \mu}{\log \log n_{k}}
$$


and

$$
\log \log \left(n_{k+1} / 2\right) \geq \log |\log | I|| \geq \log \log n_{k} .
$$

Thus if $\varepsilon>0$ and $|I|$ is sufficiently small, then

$$
t_{0}-\varepsilon \leq \frac{\log (\mu(I) /|I|)}{\log |\log | I||} \leq s_{0}+\varepsilon
$$

It follows that for all suitably small intervals,

$$
\frac{\mu(I)}{\left.|I||\log | I\right|^{s_{0}+\varepsilon}} \leq 1 \text { and } \frac{\mu(I)}{\left.|I||\log | I\right|^{t_{0}-\varepsilon}} \geq 1
$$

The bounds on the refined 1-Hausdorff co-dimension of $\mu$ follow from Corollary 5.3 .

Some of the work of Peyrière was improved by Brown et al. in [1] and Fan in [7], but significant restrictions on the dissociate set were still required. More recently, the lower bound (6.1) was improved in [8] by using the formula relating energy and the Fourier transform. It was shown there that if $\left\{n_{j}\right\}$ is any dissociate set of increasing, positive integers satisfying $\sup n_{k}{ }^{-1} \sum_{j=1}^{k-1} n_{j}<1$, then the energy dimension of $\mu_{\left\{a_{j}\right\}}=\prod_{j=1}^{\infty}(1+$ $\left.\operatorname{Re} a_{j} e^{i \pi n_{j} x}\right)$ is equal to $1-\alpha_{0}$ where

$$
\alpha_{0}=\max \left(\limsup _{k \rightarrow \infty}\left(\frac{2 \log \left|a_{k}\right|+\sum_{j=1}^{k-1} \log \left(1+\left|a_{j}\right|^{2} / 2\right)}{\log n_{k}}\right), 0\right),
$$

and the Hausdorff dimension is bounded below by

$$
\operatorname{dim}_{\mathrm{H}}\left(\mu_{\left\{a_{j}\right\}}\right) \geq 1-\limsup _{k \rightarrow \infty}\left(\frac{1}{2} \sum_{j=1}^{k}\left|a_{j}\right|^{2} / \log n_{k}\right) .
$$

This too extends to the refined dimensions.

Proposition 6.2. Suppose $\left\{n_{j}\right\}$ is a dissociate set of increasing, positive integers and assume $\sup n_{k}^{-1} \sum_{j=1}^{k-1} n_{j}<1$. Let $\left\{a_{k}\right\} \subseteq \mathbb{C},\left|a_{k}\right| \leq 1$ and suppose the Riesz product $\mu=\prod_{j=1}^{\infty}\left(1+\operatorname{Re} a_{j} e^{i \pi n_{j} x}\right)$ has energy dimension one. For $s>1$,

$$
I_{1, s}(\mu) \sim \sum_{k=1}^{\infty}\left(\log n_{k}\right)^{1-s} \frac{\left|a_{k}\right|^{2}}{2} \prod_{j=1}^{k-1}\left(1+\frac{\left|a_{j}\right|^{2}}{2}\right) .
$$

Proof. To calculate the energy of $\mu$ we should observe that if

$$
n \in \Gamma_{k} \equiv\left\{ \pm n_{k}+\sum_{j=1}^{k-1} \varepsilon_{j} n_{j}: \varepsilon_{j}=0, \pm 1\right\}
$$


then

$$
|\widehat{\mu}(n)|=\frac{\left|a_{k}\right|}{2} \prod_{j: \varepsilon_{j} \neq 0} \frac{\left|a_{j}\right|}{2}
$$

(where the empty product is one). Furthermore, if $n \in \Gamma_{k}$ then $|n| \sim n_{k}$. Thus

$$
\sum_{n \in \Gamma_{k}}(\log (n+1))^{1-s}|\widehat{\mu}(n)|^{2} \sim\left(\log n_{k}\right)^{1-s} \frac{\left|a_{k}\right|^{2}}{2} \prod_{j=1}^{k-1}\left(1+\frac{\left|a_{j}\right|^{2}}{2}\right),
$$

and therefore, provided $s>1$,

$$
I_{1, s}(\mu) \sim 1+\sum_{k=1}^{\infty}\left(\log n_{k}\right)^{1-s} \frac{\left|a_{k}\right|^{2}}{2} \prod_{j=1}^{k-1}\left(1+\frac{\left|a_{j}\right|^{2}}{2}\right) .
$$

Let us define

$$
\alpha_{0} \equiv \limsup _{k \rightarrow \infty}\left(\frac{2 \log \left|a_{k}\right|+\sum_{j=1}^{k-1} \log \left(1+\left|a_{j}\right|^{2} / 2\right)}{\log \log n_{k}}\right) .
$$

Corollary 6.3. Suppose $\alpha_{0}>0$. Then Fine $\operatorname{dim}_{\mathrm{e}}(\mu) \geq 1+\alpha_{0}$. If we assume, in addition, that $k / \log \log \left(n_{k}\right)$ is bounded, then

Fine $_{1} \operatorname{dim}_{\mathrm{H}}\left(\mu_{\left\{a_{j}\right\}}\right) \leq$ Fine $_{1} \operatorname{dim}_{\mathrm{e}}(\mu)=1+\alpha_{0}$

$$
\leq 1+\limsup _{k \rightarrow \infty}\left(\frac{1}{2} \sum_{j=1}^{k}\left|a_{j}\right|^{2} / \log \log n_{k}\right) .
$$

Proof. Clearly, the sum on the right hand side of (6.2) is infinite if infinitely many of the summands are at least one. This occurs if

$$
(1-s) \log \log n_{k}+\log \frac{\left|a_{k}\right|^{2}}{2}+\sum_{j=1}^{k-1} \log \left(1+\frac{\left|a_{j}\right|^{2}}{2}\right) \geq 0
$$

for infinitely many $k$. If $s<1+\alpha_{0}$, then this is certainly true and therefore $I_{1, s}(\mu)=\infty$ for all $s<1+\alpha_{0}$.

Conversely, the sum in (6.2) is finite if there is some $A<1$ such that for all but finitely many $k$,

$$
\left(\log n_{k}\right)^{1-s} \frac{\left|a_{k}\right|^{2}}{2} \prod_{j=1}^{k-1}\left(1+\frac{\left|a_{j}\right|^{2}}{2}\right) \leq A^{k}
$$

or, equivalently,

$$
s-1 \geq \frac{\log \left(\left|a_{k}\right|^{2} / 2\right)+\sum_{j=1}^{k-1} \log \left(1+\left|a_{j}\right|^{2} / 2\right)+k|\log A|}{\log \log n_{k}} .
$$


If we assume $k / \log \log n_{k}$ is bounded, then this occurs provided

$$
s-1 \geq \limsup _{k}\left(\frac{\log \left(\left|a_{k}\right|^{2} / 2\right)+\sum_{j=1}^{k-1} \log \left(1+\left|a_{j}\right|^{2} / 2\right)}{\log \log n_{k}}+c|\log A|\right)
$$

for some $A<1$. If $s>1+\alpha_{0}$ we can achieve this by choosing $A$ suitably close to one.

To complete the argument just note that $\log (1+x) \leq x$ for $x>0$.

Corollary 6.4. Let $\mu=\prod_{j=1}^{\infty}\left(1+\cos \pi 3^{3^{j}} x\right)$. Then Fine $_{1} \operatorname{dim}_{\mathrm{e}}(\mu)=$ $2-\ln 2 / \ln 3$.

REMARK 6.1. Similar results can obviously be proved for Riesz products

$$
\prod_{j=1}^{\infty}\left(1+\operatorname{Re} a_{j} e^{i \pi \gamma_{j} \cdot x}\right)
$$

in $\mathbb{T}^{n}$ where $\left\{\gamma_{j}\right\} \subseteq \mathbb{Z}^{n}$ is dissociate and satisfies $\sum_{j=1}^{k-1}\left|\gamma_{j}\right| \leq c\left|\gamma_{k}\right|$ for some $c<1$.

\section{References}

[1] G. Brown, W. Moran and C. Pearce, Riesz products, Hausdorff dimension and normal numbers, Math. Proc. Cambridge Philos. Soc. 101 (1987), 529-540.

[2] C. Carathéodory, Über das lineare Mass von Punktmengen - eine Verallgemeinerung des Längenbegriffs, Nachr. Königl. Ges. Wiss. Göttingen Math.-Phys. Kl. 1914, 404426.

[3] I. Chavel, Eigenvalues in Riemannian Geometry, Pure Appl. Math. 115, Academic Press, Orlando, 1984.

[4] E. B. Davies, Heat Kernels and Spectral Theory, Cambridge Univ. Press, Cambridge, 1989.

[5] K. Falconer, Fractal Geometry. Mathematical Foundations and Applications, Wiley, Chichester, 1990.

[6] —, Techniques in Fractal Geometry, Wiley, Chichester, 1997.

[7] A. H. Fan, Quelques propriétés des produits de Riesz, Bull. Sci. Math. 117 (1993), 421-439.

[8] K. E. Hare and M. Roginskaya, A Fourier series formula for energy of measures with applications to Riesz products, Proc. Amer. Math. Soc. 131 (2003), 165-174.

[9] - - - Multipliers of spherical harmonics and energy of measures on the sphere, Ark. Mat., to appear.

[10] —, - , The energy of signed measures, Proc. Amer. Math. Soc., to appear.

[11] K. E. Hare, D. C. Wilson and W.-L. Yee, Pointwise estimates of the size of characters of compact Lie groups, J. Austral. Math. Soc. Ser. A 69 (2000), 61-84.

[12] K. E. Hare and K. Yeats, The size of characters of exceptional Lie groups, J. Austral. Math. Soc., to appear.

[13] S. Helgason, Groups and Geometric Analysis. Integral Geometry, Invariant Differential Operators and Spherical Functions, Pure Appl. Math. 113, Academic Press, Orlando, 1984. 
[14] Y. Kannai, Short time asymptotic behavior for parabolic equations, in: Analytical and Numerical Approaches to Asymptotic Problems in Analysis (Nijmegen, 1980), O. Axelsson, L. S. Frank and A. van der Sluis (eds.), North-Holland Math. Stud. 47, North-Holland, Amsterdam, 1981, 117-132.

[15] P. Li and S.-T. Yau, On the parabolic kernel of the Schrödinger operator, Acta Math. 156 (1986), 153-201.

[16] P. Mattila, Geometry of Sets and Measures in Euclidean Spaces, Cambridge Stud. Adv. Math. 44, Cambridge Univ. Press, Cambridge, 1995.

[17] J. Neveu, Mathematical Foundations of the Calculus of Probability, Holden-Day, San Francisco, 1965.

[18] P. Petersen, Riemannian Geometry, Springer, New York, 1998.

[19] J. Peyrière, Étude de quelques propriétés des produits de Riesz, Ann. Inst. Fourier (Grenoble) 25 (1975), 127-169.

[20] C. Rogers, Hausdorff Measures, Cambridge Univ. Press, London, 1970.

Department of Pure Mathematics

University of Waterloo

Waterloo, Ontario, N2L 3G1, Canada

E-mail: kehare@uwaterloo.ca
Department of Mathematics Chalmers TH \& Göteborg University Eklandagatan 86

SE 412 96, Göteborg, Sweden E-mail: maria@math.chalmers.se

Received November 26, 2002

Revised version June 26, 2003 